\title{
A Generalized Prefix Construction for OFDM Systems over Quasi-Static Channels
}

\author{
Todor Cooklev* $\quad$ Hakan Doğan ${ }^{\dagger} \quad$ Renato J. Cintra ${ }^{\ddagger}$ Hakan Yıldız
}

\begin{abstract}
All practical OFDM systems require a prefix to eliminate inter-symbol interference at the receiver. Cyclic prefix (CP) and zero-padding are well-known prefix construction methods, the former being the most employed technique in practice due to its lower complexity. In this paper we construct an OFDM system with a generalized CP. It is shown that the proposed generalized prefix effectively makes the channel experienced by the packet different from the actual channel. Using an optimization procedure, lower bit error rates can be achieved, outperforming other prefix construction techniques. At the same time the complexity of the technique is comparable to the CP method. The presented simulation results show that the proposed technique not only outperforms the CP method, but is also more robust in the presence of channel estimation errors and mobility. The proposed method is appropriate for practical OFDM systems.
\end{abstract}

\section{Keywords}

Generalized prefix, generalized skew-circular convolution, orthogonal frequency-division multiplexing (OFDM), deep fading channels.

\section{INTRODUCTION}

Bandlimited wireless communication channels exhibit significant variations in gain and phase along different frequencies. This phenomenon reduces the capacity of the channel. In the case of orthogonal frequencydivision multiplexing (OFDM) systems, associated subcarriers can experience deep fades. In the extreme case these deep fades may become spectral nulls. When a cyclic prefix $(\mathrm{CP})$ is used, these spectral nulls are known to significantly limit the bit error rate performance.

In literature, there are several approaches to deal with the deep fade problem. Practical OFDM systems [1] simply accept this difficulty, and try to minimize it by the usage of forward error correction (FEC) techniques. However, applying FEC increases complexity and overhead, and it may not be the right tool for this task [2]. In fact, several signal processing methods have been introduced that, without using FEC, improve the performance in channels with spectral nulls. One such method is the precoded OFDM, which inserts one zero between every two information symbols [3]. Its disadvantage is the reduction of data rate by one-third.

\footnotetext{
*Todor Cooklev is with the Wireless Technology Center, Indiana University-Purdue University Fort Wayne (IPFW), USA (e-mail: cooklevt@ipfw.edu).

${ }^{\dagger}$ Hakan Doğan is with the Department of Electrical and Electronics Engineering, Istanbul University,34320, Avcilar, Istanbul, Turkey (e-mail: hdogan@istanbul.edu.tr).

${ }^{\ddagger}$ Renato J. Cintra is with the Departamento de Estatística, Universidade Federal de Pernambuco, Recife, Brazil (e-mail: rjdsc@stat.ufpe.org).

${ }^{\S}$ Hakan Ylldız is with the Ericsson, 34398, Maslak, Istanbul, Turkey (e-mail: hakan.yildiz@ericsson.com).
} 
In addition, precoded OFDM requires the wireless channel to be static for the duration of multiple OFDM symbols. This requirement may force the duration of the OFDM block to be shorter than the channel coherence time.

Another approach is to replace the commonly used CP with zero-padding (ZP) 4, 5]. Such a technique guarantees that the vector of information symbols can be recovered regardless of the presence of spectral nulls [4,5]. The reason is that the equivalent channel matrix is invertible even if there are spectral nulls. However, ZP has disadvantages. In particular, its associated channel matrix is not circulant and cannot be diagonalized by the discrete Fourier transform (DFT), which is generally computed via a fast Fourier transform (FFT) algorithm [6]. As a result, $\mathrm{ZP}$ is substantially more complex than $\mathrm{CP}$ since it requires a matrix inversion for every packet. Low-complexity ZP schemes have been developed, which however are suboptimal [5]. Zero-padding also proves to be ineffective in terms of timing and frequency synchronization [7]. As a result, neither precoding, nor ZP are techniques employed in practical systems, such as the IEEE 802 standards [1]. Indeed, all practical systems use a CP because of its computational simplicity and convenience for detection and synchronization.

In [8] Muck et al. proposed the insertion of a pseudo-random postfix (PRP) between OFDM symbols. This postfix is constructed by means of a known vector weighted by a pseudo-random scalar sequence to enable semi-blind channel estimation. As described in [8], at the receiver, PRP-OFDM symbols are transformed into ZP-OFDM symbols by subtracting the contribution of the postfix. Subsequent additional processing is performed to obtain a symbol corresponding to CP-OFDM.

Other approaches that have been suggested are based on the idea that the CP can be replaced by a pre-defined sequence of known symbols [9, 10. These approaches are referred to as known symbol padding (KSP) OFDM and unique word (UW) OFDM [11. In these systems, the known samples in the guard interval could be used for channel, timing and carrier offset estimation.

UW-OFDM is a fundamentally different technique, which introduces correlation among the subcarriers and the UW is part of the DFT interval, whereas the prefix in CP-OFDM is added after the DFT. This makes comparisons under identical assumptions difficult. For example, the design of UW is considered subject to a constraint on the peak-to-average power ratio (PAPR) [12. In KSP-OFDM, the KSP part is not part of DFT, similar to CP-OFDM, but performance simulations reported in [13] show that KSP-OFDM suffers from noise enhancement and has a slightly worse performance than CP-OFDM.

Our work is different: we use a prefix like the CP, but weighted by a complex number. This new prefix can be referred to as a generalized prefix. The proposed technique effectively changes the phases of the multipath components. We show that if the prefix is appropriately constructed the technique can effectively transform the wireless channel experienced by the OFDM symbols into a higher-capacity channel, leading to a lower bit-error rate. The described prefix can convert a channel with spectral nulls or deep fades into a channel with no spectral nulls or fades that are less deep. Furthermore, if all other parameters are identical, it outperforms several prefix construction techniques at the cost of a minor increase in the computational complexity. We present extensive simulation results. The proposed technique outperforms the CP method, particularly in channels with long delay spreads that are characterized by deep fades. Furthermore, the proposed system is more robust to channel estimation errors, i.e. compared with CP-OFDM the margin of improvement is even higher in the presence of channel estimation errors.

The organization of the paper is as follows. In Section 2 we formulate the problem mathematically. A 
generalized prefix is introduced and discussed in Section 3. Next we detail how the proposed generalized prefix can be optimized and the associated computational complexity is analyzed in Section 4 Section 5 contains the simulation results and concluding remarks are drawn in Section 6 .

\section{Signal Model}

We consider a conventional OFDM system with single transmitting and receiving antennas. The complex information symbols, referred to as subcarriers, are denoted by $X[k], k=0,1, \ldots, N-1$, where $N$ is the number of subcarriers. These complex symbols are obtained from the information bits via a bit-tosymbol mapping such as quadrature phase-shift keying, or more generally $M$-ary quadrature amplitude modulation (QAM). Without any limitation to the proposed methodology, hereafter we assume that the QAM scheme is employed.

An application of the inverse DFT generates a new signal $\mathbf{x}$ that combines all subcarriers:

$$
\mathbf{x}=\mathbf{F}_{N}^{*} \cdot \mathbf{X}
$$

where $\mathbf{F}_{N}$ is the $N$-point DFT matrix which is defined as $\mathbf{F}_{N} \triangleq[\exp (-2 \pi i k / N)]_{i, k=0, \ldots, N-1}$, superscript * denotes Hermitian conjugation, and $\mathbf{X}=[X[0], X[1], \ldots, X[N-1]]^{t}$. Next, cyclic prefix (CP) of length $K$ is added to the OFDM symbols $\mathbf{x}$ to obtain a new vector $\widetilde{\mathbf{x}}$ :

$$
\widetilde{\mathbf{x}} \triangleq[x[N-K], x[N-K-1], \ldots, x[N-1], x[0], x[1], \ldots, x[N-1]]^{t},
$$

which has $N+K$ components.

A wireless channel with $L$ multipath components can be modeled with respect to the baseband by an $L$-point FIR filter, whose transfer function is

$$
H(z)=h[0]+h[1] z^{-1}+\cdots+h[L-1] z^{-L+1} .
$$

The CP prevents ISI, as long as its length exceeds the duration of the impulse response of the channel, or $K \geq L-1$. Then the received signal is a linear convolution between the transmitted signal and the channel impulse response for $m=0,1, \ldots, N+K+L-2$ :

$$
y[m]=\sum_{l=0}^{L-1} h[l] \widetilde{x}[m-l]+w[m],
$$

where $w[m]$ is Gaussian noise vector with zero mean and variance $\sigma^{2}=N_{0} / 2$ and $N_{0}$ is the single-sided power spectral density. Over the first $K$ received symbols there is inter-symbol interference, and these symbols are ignored. The receiver processes $y[m]$ only for $m=K, K+1, \ldots, N+K-1$, or alternatively

$$
y[m]=\sum_{l=0}^{L-1} h[l] x\left[\langle m-K-l\rangle_{N}\right]+w[m],
$$

where $\langle\cdot\rangle_{N}$ represents the modulo $N$ operation. The CP removal not only eliminates the ISI, but also 


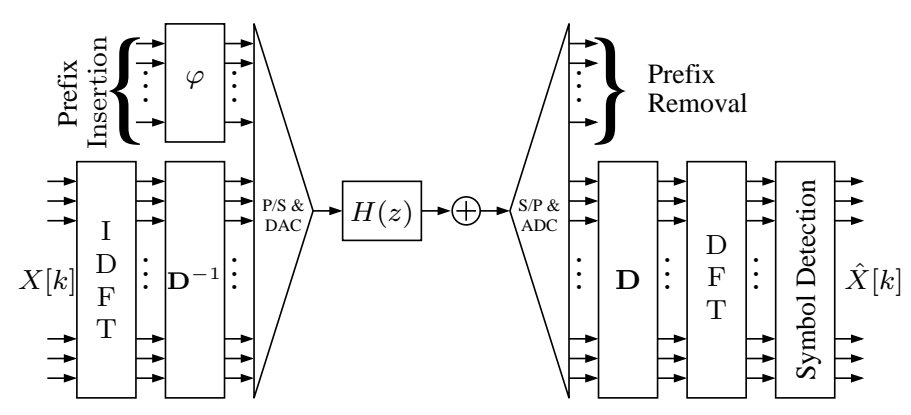

Figure 1: Proposed OFDM system.

converts the linear convolution with the channel impulse response into a cyclic convolution. Consider the $N$ point signal $\mathbf{y}=[y[K], y[K+1], \ldots, y[N+K-1]]^{t}$ and the vector of channel impulse response components $\mathbf{h}=[h[0], h[1], \ldots, h[L-1], 0, \ldots, 0]^{t}$ padded with an appropriate number of zeros, so that its length becomes $N$. By taking (10) into account, the referred cyclic convolution can be expressed in matrix terms according to

$$
\mathbf{y}=\mathbf{H} \cdot \mathbf{F}_{N}^{*} \cdot \mathbf{X}+\mathbf{w}
$$

where $\mathbf{w}=[w[K], w[K+1], \ldots, w[N+K-1]]^{t}$ and $\mathbf{H}$ is the circulant matrix whose first column is the vector $\mathbf{h}$ :

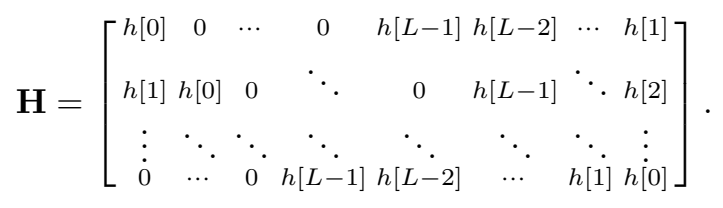

It is assumed that the channel is characterized by slow fading, i.e. the channel impulse response does not change within one OFDM symbol duration. At the receiver shown in Fig. 1 the forward DFT is applied to obtain

$$
\mathbf{Y}=\mathbf{F}_{N} \cdot \mathbf{y}=\mathbf{F}_{N} \cdot \mathbf{H} \cdot \mathbf{F}_{N}^{*} \cdot \mathbf{X}+\mathbf{F}_{N} \cdot \mathbf{w}
$$

The information symbol vector $\mathbf{X}$ must be recovered from $\mathbf{Y}$. This can be accomplished by the multiplication of $\mathbf{Y}$ with the inverse of $\mathbf{F}_{N} \cdot \mathbf{H} \cdot \mathbf{F}_{N}^{*}$. This matrix inversion is greatly simplified numerically, because the DFT diagonalizes circulant matrices:

$$
\begin{aligned}
\mathbf{F}_{N} \cdot \mathbf{H} \cdot \mathbf{F}_{N}^{*} & =\operatorname{diag}(H[0], H[1], \ldots, H[N-1]) \\
& =\operatorname{diag}\left(\mathbf{F}_{N} \cdot \mathbf{h}\right),
\end{aligned}
$$

where $\operatorname{diag}(\cdot) \operatorname{returns}$ a diagonal matrix and the sequence $H[k], k=0,1, \ldots, N-1$, is the $N$-point DFT of the channel impulse response.

The diagonalization operation effectively decomposes the channel into parallel and ISI-free sub-channels. In other words, the frequency-selective channel is transformed into a channel with flat fading per subcarrier. As a result, a simple zero-forcing (ZF) detector can be employed to obtain an estimate $\widehat{\mathbf{X}}$ of the transmitted 
information sequence. Requiring only one division operation per subcarrier, the elements of $\widehat{\mathbf{X}}$ are given by

$$
\widehat{X}[k]=\frac{Y[k]}{H[k]}, \quad k=0,1, \ldots, N-1 .
$$

The main disadvantage of the described OFDM system is the performance in channels with spectral nulls. Indeed, if one or more of the quantities $H[k], k=0,1, \ldots, N-1$, are equal to zero, then (10) yields to an indetermination. Equivalently, the diagonal matrix $\operatorname{diag}\left(\mathbf{F}_{N} \cdot \mathbf{h}\right)$ is not invertible.

Let the probability of bit error for the chosen QAM bit-to-symbol mapping scheme in an AWGN channel be $P_{\mathrm{QAM}}\left(E_{b} / N_{0}\right)$, where $E_{b}$ is the energy per bit. Then the probability of bit error $P_{e}$ of the OFDM system with cyclic prefix is 3 :

$$
P_{e}=\frac{1}{N} \sum_{k=0}^{N-1} P_{\mathrm{QAM}}\left(\frac{N}{N+K} \frac{E_{b}}{N_{0}}|H[k]|^{2}\right) .
$$

Notice that the quantity $\frac{N}{N+K} \frac{E_{b}}{N_{0}}$ is a constant.

If the channel has one spectral null, or $H[k]=0$ for a given subcarrier $k$, then the symbol transmitted on that subcarrier has a probability of error equal to $1 / 2$, regardless of the signal-to-noise ratio. Since the probability of error of the entire system is the average of the probabilities of error for each of the subcarriers, it is known that the subcarriers with the largest probability of error dominate the overall system error probability. Since there are $N$ subcarriers, the probability of error will always be higher than $P_{e} \geq 1 /(2 N)$. In other words, there is an error floor for high signal-to-noise values, or equivalently, there is a loss in frequency diversity [4. The effect of deep fades will be similar, only less severe. This is a significant disadvantage.

As mentioned in the introduction, ZP is another possible prefix construction technique. After the receiver discards all received samples, except those from $K$ to $N+K-1$, ZP leads to a received signal as described in (6) with the equivalent channel matrix being equal to:

$$
\mathbf{H}_{\mathrm{ZP}}=\left[\begin{array}{cccccccc}
h[0] & 0 & \cdots & 0 & 0 & \cdots & 0 & 0 \\
h[1] & h[0] & 0 & \ddots & \ddots & \ddots & \ddots & 0 \\
\vdots & \ddots & \ddots & \ddots & \ddots & \ddots & \ddots & \vdots \\
0 & \cdots & 0 & h[L-1] & h[L-2] & \cdots & h[1] & h[0] \\
0 & \cdots & 0 & 0 & h[L-1] & \cdots & h[2] & h[1] \\
\vdots & \vdots & \vdots & \vdots & \vdots & \vdots & \vdots & \vdots
\end{array}\right] .
$$

This matrix is similar to $\mathbf{H}$; however it is not necessarily square. Indeed, it is a tall channel matrix.

The matrix $\mathbf{H}_{\mathrm{ZP}}$ cannot be diagonalized by applying the forward DFT. As a result, the receiver has to perform an $N \times N$ matrix inversion for every OFDM symbol to obtain an estimate $\widehat{\mathbf{X}}$ of the information symbol vector:

$$
\widehat{\mathbf{X}}=\mathbf{F}_{N} \cdot \mathbf{H}_{\mathrm{ZP}}^{+} \cdot \mathbf{y}=\mathbf{X}+\mathbf{F}_{N} \cdot \mathbf{H}_{\mathrm{ZP}}^{+} \cdot \mathbf{w}
$$

where superscript ${ }^{+}$denotes the pseudoinverse operation.

The matrix $\mathbf{H}_{\mathrm{ZP}}$ is always full column rank - even in the presence of spectral nulls - as long as $h[0] \neq 0$, which is a reasonable assumption. However, still the ZP method is not used by any practical system due to its high computational complexity. 


\section{Proposed Generalized Prefix}

In the proposed system the prefix is constructed by multiplying the last $K$ samples with a complex number $\varphi \neq 0$ and appending them to $\mathbf{x}$ :

$$
\widetilde{\mathbf{x}}=[\varphi x[N-K], \varphi x[N-K-1], \ldots, \varphi x[N-1], x[0], x[1], \ldots, x[N-1]]
$$

Just like in the case for $\mathrm{CP}$, the receiver processes $y[m]$ only for $m=K, K+1, \ldots, N+K-1$. The received signal can be expressed as

$$
y[m]=\sum_{l=0}^{L-1} h[l] u[m-K-l] x\left[\langle m-K-l\rangle_{N}\right]+w[m],
$$

where the sequence $u[\cdot]$ is

$$
u[n]= \begin{cases}1, & \text { if } n \geq 0 \\ \varphi, & \text { if } n<0 .\end{cases}
$$

It is worth pointing out that (15) has not been defined as a convolution operation in the signal processing literature [14 16]. The special cases $\varphi=1$ and $\varphi=-1$ correspond to circular and skew-circular convolution [15,16. We suggest to call the particular type of convolution described in (15) as generalized skew-circular convolution.

Considering the proposed prefix, the equivalent channel matrix is given by:

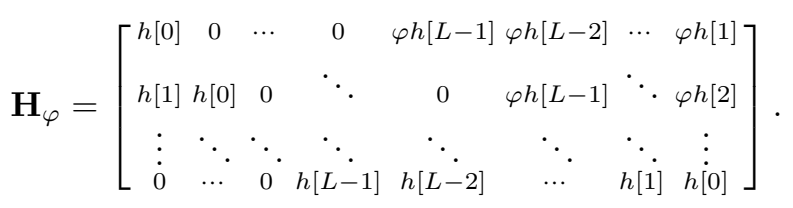

The matrix $\mathbf{H}_{\varphi}$ can be diagonalized. Let us denote the elements of $\mathbf{H}_{\varphi}$ as $h_{i j}^{\varphi}, i, j=1,2, \ldots, N$. We note that $\mathbf{H}_{\varphi}$ is a Toeplitz matrix with the property $h_{i j}^{\varphi}=\varphi h[N+i-j]$ for $i-j<0$. For $i-j \geq 0, \mathbf{H}_{\varphi}$ is constructed as $\mathbf{H}$. We suggest that this matrix type is referred to as generalized skew-circular, following the name of the convolution operation. The following result allows the use of the proposed prefix in OFDM systems.

Theorem 1 If $\psi$ is a complex number for which

$$
\psi^{N}=\varphi,
$$

then

$$
\mathbf{F}_{N} \cdot \mathbf{D} \cdot \mathbf{H}_{\varphi} \cdot \mathbf{D}^{-1} \cdot \mathbf{F}_{N}^{*}=\operatorname{diag}\left(H_{\psi}[0], H_{\psi}[1], \ldots, H_{\psi}[N-1]\right)=\operatorname{diag}\left(\mathbf{F}_{N} \cdot \mathbf{D} \cdot \mathbf{h}\right),
$$

where $H_{\psi}[k], k=0,1, \ldots, N-1$ is the DFT of the vector $\mathbf{D} \cdot \mathbf{h}$, and

$$
\mathbf{D}=\operatorname{diag}\left(1, \psi, \psi^{2}, \ldots, \psi^{N-1}\right)
$$


Proof: Suppose that the $(i, j)$ entry of $\widetilde{\mathbf{H}}=\mathbf{D} \cdot \mathbf{H}_{\varphi} \cdot \mathbf{D}^{-1}$ is denoted by $\widetilde{h}_{i j}$. Considering the DFT property in (9), the above result can be proven by establishing that $\mathbf{D} \cdot \mathbf{H}_{\varphi} \cdot \mathbf{D}^{-1}$ is a circulant matrix. Circulant matrices have the property $\widetilde{h}_{i j}=\widetilde{h}_{k l}$ when $k-l=\langle i-j\rangle_{N}$. Thus, the following holds:

$$
\begin{aligned}
\widetilde{h}_{i j} & = \begin{cases}\psi^{i-1} h[i-j] \psi^{-j+1}, & \text { if } i-j \geq 0, \\
\psi^{i-1} \psi^{N} h[N+i-j] \psi^{-j+1}, & \text { if } i-j<0\end{cases} \\
& = \begin{cases}\psi^{i-j} h[i-j], & \text { if } i-j \geq 0, \\
\psi^{N+i-j} h[N+i-j], & \text { if } i-j<0 .\end{cases}
\end{aligned}
$$

Considering (22) and $h_{i j}^{\varphi}=\varphi h[N+i-j]$ for $i-j<0$, if $k-l=\langle i-j\rangle_{N}$, then it follows that $\widetilde{h}_{i j}=\widetilde{h}_{k l}$ . Therefore the matrix $\widetilde{\mathbf{H}}$ is circulant. Additionally, from (21) we notice that the first column of $\widetilde{\mathbf{H}}$ is the vector $\mathbf{D} \cdot \mathbf{h}$ :

$$
\widetilde{\mathbf{H}}=\left[\begin{array}{cccccccc}
h[0] & 0 & \cdots & 0 & \psi^{L-1} h[L-1] & \psi^{L-2} h[L-2] & \cdots & \psi h[1] \\
\psi h[1] & h[0] & 0 & \ddots & 0 & \psi^{L-1} h[L-1] & \ddots & \psi^{2} h[2] \\
\vdots & \ddots & \ddots & \ddots & \ddots & \ddots & \ddots & \vdots \\
0 & \cdots & 0 & \psi^{L-1} h[L-1] & \psi^{L-2} h[L-2] & \cdots & \psi h[1] & h[0]
\end{array}\right] .
$$

A comparable result was proven in [8] for the postfix OFDM.

The block diagram of the proposed system is shown in Fig. 1. Multicarrier modulation is performed by

$$
\mathbf{x}=\mathbf{D}^{-1} \cdot \mathbf{F}_{N}^{*} \cdot \mathbf{X}
$$

At the receiver side the signal is multiplied by $\mathbf{D}$, and then the DFT is applied to obtain

$$
\begin{aligned}
\mathbf{Y} & =\mathbf{F}_{N} \cdot \mathbf{D} \cdot \mathbf{H}_{\varphi} \cdot \mathbf{D}^{-1} \cdot \mathbf{F}_{N}^{*} \cdot \mathbf{X}+\mathbf{F}_{N} \cdot \mathbf{D} \cdot \mathbf{w} \\
& =\operatorname{diag}\left(H_{\psi}[0], H_{\psi}[1], \ldots, H_{\psi}[N-1]\right) \cdot \mathbf{X}+\mathbf{F}_{N} \cdot \mathbf{D} \cdot \mathbf{w} .
\end{aligned}
$$

Since $\mathbf{D}$ is a diagonal matrix, vector multiplications by $\mathbf{D}$ or $\mathbf{D}^{-1}$ require only $N$ complex multiplications. Therefore the arithmetic complexity of modulation/demodulation operation is comparable to the CP case. On the other hand, the proposed prefix requires an optimization procedure to find the optimal value of $\psi$; this also requires an additional complexity.

In general, the quantities $\varphi$ and $\psi$ can be any non-null complex numbers that satisfy (18). However, if $|\varphi| \neq 1$, then the absolute values of the elements of $\mathbf{D}$ and $\mathbf{D}^{-1}$ are either very large or very small. This fact makes the system prone to numerical instability issues. Additionally, the PAPR is increased. To maintain the same PAPR, it is required that $|\varphi|=1$. This choice of $\varphi$ also implies $|\psi|=1$.

\section{Generalized Prefix}

The proposed prefix construction transforms the wireless channel $\mathbf{h}$ experienced by the system into an equivalent channel with impulse response equal to the product $\mathbf{D} \cdot \mathbf{h}$, whose transfer function is given by:

$$
H_{\psi}(z)=h[0]+\psi h[1] z^{-1}+\psi^{2} h[2] z^{-2}+\cdots+\psi^{L-1} h[L-1] z^{-(L-1)} .
$$


It is now clear that the generalized prefix has a physical meaning: the $n$th multipath component is multiplied by $\psi^{n}$. By adopting $|\psi|=1$, we can write $\psi=e^{j \alpha}$, for $\alpha \in[0,2 \pi]$; thus only the phase of the multipath components is affected.

The role of $\psi$ is better understood in terms of the frequency response $H_{\psi}\left(e^{j \omega}\right)$ of the equivalent channel, which can be related to the frequency response $H\left(e^{j \omega}\right)$ of the actual channel:

$$
H_{\psi}\left(e^{j \omega}\right)=\sum_{n=0}^{N-1} \psi^{n} h[n] e^{-j \omega n}=H\left(e^{j(\omega-\alpha)}\right),
$$

since $\psi=e^{j \alpha}$. Thus, the proposed prefix construction represents a shift in frequency domain of the channel frequency response.

Since the subcarriers are centered at the discrete frequencies $\omega_{k}=2 \pi k / N, k=0,1, \ldots, N-1$, it is important to characterize the associated quantities $H[k]=H\left(e^{j \omega_{k}}\right)$. Suppose that the channel frequency response has a null at $\omega_{k}$, for some $k$. In this case, the frequency response of the channel, actually experienced by the system can avoid this situation by shifting the spectral null away from the location $\omega_{k}$.

It must be noted that $\varphi=\psi^{N}=1$ or $\varphi=\psi^{N}=-1$, which correspond to circular or skew-circular convolution, respectively, may not be adequate choices. In fact, this selection of $\varphi$ entails $\psi=e^{j 2 \pi / N}$ or $\psi=e^{j \pi / N}$. Thus, the implied shift in frequency is $\alpha=2 \pi / N$ or $\alpha=\pi / N$, respectively. Since the subcarriers are exactly $2 \pi / N \mathrm{rad} /$ samples apart from each other, the spectral shift generated by circular or skew-circular convolution may simply move an existing zero of the sampled transfer function from one subcarrier to another. This would not be effective. In order to appropriately avoid spectral nulls, it is necessary to have a greater freedom of choice for $\psi$.

The distinct advantage of the generalized prefix is that for certain values of $\psi$ the wireless channel experienced by the transmitted OFDM symbols can lead to a lower bit error rate. In principle, any choice of $\alpha \neq 2 \pi m / N$, where $m$ is an integer, is likely to improve the performance of the above described multicarrier modulation system up to certain extent. Even a random selection scheme for the value of $\psi$ could represent an enhancement in avoiding spectral nulls. However, there exists an optimal choice of $\psi$ that leads to the lowest bit error rate, therefore increasing system performance. Next we discuss two methods of finding the optimal value of $\psi$.

\subsection{Minimal Probability of Error Approach}

In the first approach, we consider the probability of bit error the figure of merit to be optimized. Thus, the minimization problem is:

$$
\alpha^{*}=\left.\arg \min _{\alpha \in\left[0, \frac{2 \pi}{N}\right]} P_{e}(\psi)\right|_{\psi=e^{j \alpha}}
$$

where $\alpha^{*}$ is the optimum frequency shift and $P_{e}(\psi)$ is the bit error probability of the proposed system given by

$$
P_{e}(\psi)=\frac{1}{N} \sum_{k=0}^{N-1} P_{\mathrm{QAM}}\left(\frac{N}{N+K} \frac{E_{b}}{N_{0}}\left|H_{\psi}[k]\right|^{2}\right) .
$$


Input: Initial interval $[a, b]$; tolerance $\epsilon$; objective function $P_{e}(\cdot)$.

Output: Optimum frequency shift $\alpha^{*}$.

Method: Golden section search.

1: $\Phi \leftarrow(\sqrt{5}-1) / 2$

2: $p \leftarrow b-(b-a) \Phi$

3: $q \leftarrow a+(b-a) \Phi$

4: $f_{p} \leftarrow P_{e}\left(e^{j p}\right)$

5: $f_{q} \leftarrow P_{e}\left(e^{j q}\right)$

6: while $b-a \geq \epsilon$ do

if $f_{p} \leq f_{q}$ then

$b \leftarrow q$

$q \leftarrow p$

$p \leftarrow b-(b-a) \Phi$

$f_{q} \leftarrow f_{p}$

else

$f_{p} \leftarrow P_{e}\left(e^{j p}\right)$

$a \leftarrow p$

$p \leftarrow q$

$q \leftarrow a+(b-a) \Phi$

$f_{p} \leftarrow f_{q}$

$f_{q} \leftarrow P_{e}\left(e^{j q}\right)$

end if

end while

21: return $\alpha^{*} \leftarrow(a+b) / 2$

Figure 2: Minimization algorithm: golden section search.

The above equation is analogous to (11). We assume that $P_{e}(\cdot)$ is continuous and unimodal over the search space for a fixed $E_{b} / N_{0}$. Additionally, $P_{e}(\cdot)$ is a single-variable bounded nonlinear function. Because the frequency separation between adjacent subcarriers is $2 \pi / N$ the search space can be $[0,2 \pi / N]$, and the set of frequency shifts $\left\{\alpha^{*}+2 \pi m / N\right\}$, where $m$ is an integer, constitutes a class of equivalence.

Under the above conditions, the suggested minimization problem can be solved without resorting to derivatives by means of numerical techniques such as the golden section search method. Let us assume that $[a, b]$ is a given interval where the sought minimum is located. In this case, the points $p$ and $q$ can be calculated as follows:

$$
\begin{aligned}
& p=b-(b-a) \Phi, \\
& q=a+(b-a) \Phi,
\end{aligned}
$$

where $\Phi=\frac{\sqrt{5}-1}{2}$ is the golden ratio conjugate. After evaluating the function $P_{e}(\cdot)$ at points $p$ and $q$, a new search interval is established according to whether $P_{e}\left(e^{j p}\right) \leq P_{e}\left(e^{j q}\right)$ or not [17. The algorithm is summarized in Fig. 2,

\subsection{MaX-Min Approach}

Searching for the optimal value of $\alpha$ by solving the optimization problem as described in (27) involves several calls to the $Q$-function and other arithmetical operations. Also the function $P_{e}(\cdot)$ depends on the chosen modulation order. 
Instead of (27), as an alternative, we can consider the following optimization problem:

$$
\alpha^{*}=\max _{\alpha \in\left[0, \frac{2 \pi}{N}\right]} \min _{k}\left|H_{\psi}[k]\right|_{\psi=e^{j \alpha}} .
$$

Intuitively, the above problem returns the value of $\alpha$ (therefore, $\psi$ ) that makes the minimum values of $H_{\psi}[k]$ (deep fades) to be as large as possible.

Our simulations indicate that solving the above minimax type optimization problem [18] leads to the same numerical results as the optimization technique in from (27). The discussed above golden search method is also employed in this procedure.

\subsection{Practical Considerations}

An important feature of the proposed technique is that the optimal value can be determined not only at the transmitter (if the transmitter has channel state information (CSI)), but also at the receiver. Although making CSI available at the transmitter is difficult for both time division duplex and frequency division duplex modes [19] recent wireless systems such as 802.11 can provide CSI at the transmitter [1].

Still, a considerable amount of information must be sent back to the transmitter, especially for mobile systems. Therefore, we propose that $\psi$ is calculated at the receiver and only the value of $\psi$ is sent to the transmitter. In this case, feedback can be provided with minimum overhead and very quickly to match the channel variability.

\subsection{Computational Complexity}

In the proposed system the transmitter is required to compute $2 N+K$ complex multiplications (CMs): $K$ CMs to generate the prefix in (14); $N$ CMs for $\mathbf{D}^{-1}$; and $N$ CMs to perform multiplication by $\mathbf{D}^{-1}$ according to (20). At the receiver, the diagonal matrix $\mathbf{D}$ must also be obtained from the value of $\psi$ and the received signal must be multiplied by $\mathbf{D}$; each of these operations requires $N$ CMs. Now let us evaluate the complexity of finding the optimal value of $\psi$.

\subsubsection{Probability Based Optimization Approach}

First we analyze the method described in (27). To find the needed optimal $\psi$ for $\mathbf{D}$, the receiver calculates the associated cost function by using (25) and (28) in the golden search algorithm. We assume that this optimization algorithm takes $Z$ iterations to converge. Thus, for each iteration, we have the following computational complexities.

The evaluation of (25) requires only $L-1 \mathrm{CMs}$. An $N$-point DFT call is required to provide the magnitude of the frequency response of the equivalent channel as shown in (28). The multiplicative complexity of the DFT is in $\mathcal{O}\left(N \log _{2} N\right)$. The evaluation of function $P_{\mathrm{QAM}}$ in (28) requires the $Q$-function [20]. Assuming that the $Q$-function values are stored in a lookup table, (28) requires $N$ real multiplications by a constant in the argument of $P_{\mathrm{QAM}}$ and an $N$-point summation.

Therefore, the proposed prefix based system increases the computational complexity by $2 N+K+2 N+$ $Z \times\left(N+L-1+N \log _{2} N\right)$ CMs. 


\subsubsection{MaX-Min Optimization Approach}

On the other hand, the described max-min method as shown in (30) is computationally less expensive. Indeed, in comparison to (27), it eliminates (i) the evaluation $P_{\mathrm{QAM}}$; (ii) the $N$-point summation; and (iii) the required multiplications by constants. Since the function $P_{\mathrm{QAM}}$ is not needed, it also does not require any sort of lookup table.

It requires (i) the evaluation of (25) $(L-1 \mathrm{CMs})$; (ii) a DFT call; and (iii) the identification of the smallest element in a vector. The DFT computation is in $\mathcal{O}\left(N \log _{2} N\right)$. Finding the minimum in a vector is comparatively a simple operation [21] (cf. (27)). Thus, we have a total multiplicative complexity of $2 N+K+2 N+Z\left(L-1+N \log _{2} N\right)$. Due to its simplicity, the max-min method may be regarded as computationally faster than the first approach.

For both methods, the above detailed estimates of the number of multiplications are worst case scenarios. This is due to the fact that we are assuming that the DFT computation would require exactly $N \log _{2} N$ multiplications, which is an upper bound. For example, assuming $N=512$ and using the Rader-Brenner radix-two fast Fourier transform [6], the DFT can be computed in just 3076 real multiplications, which is well below $512 \times \log _{2} 512=4608 \mathrm{CMs}$ (13824 real multiplications) [6].

For $N \gg L$ and $N \gg K$, as is the case in practical systems, the computational complexity is $\mathcal{O}(N)$ at the transmitter. At the receiver side, we have an asymptotical computational multiplicative complexity in $\mathcal{O}(Z N \log N)$ for both methods.

Overall the computational complexity of the proposed method is higher than the complexity of CPOFDM for both optimization techniques. However, $\psi$ can be calculated at the beginning and can be used while the channel is assumed to be static. Therefore, we can say that at the expense of a minor increase in the computational complexity we can achieve a performance gain which will be shown in the simulation section.

\section{Simulation Results}

In this section, Monte Carlo simulations of data transmission are used to analyze the average BER performance of the proposed generalized prefix for two different scenarios. For each Monte Carlo run, a channel is generated according to the simulation environment described in its respective scenario. The first scenario is a simple example to illustrate the effectiveness of the proposed technique. The proposed prefix in this case transforms the linear convolution into a skew-circular convolution. In a second scenario, we present results obtained from a practical example, where random multipath channels were optimized. In our simulations, we selected the tolerance factor for the golden section search as $\epsilon=10^{-3}$. In this case, the golden search algorithm required less than 10 iterations to find an optimal value $\psi=e^{j \alpha}$ in the search space $\alpha \in[0,2 \pi / N]$.

\subsection{Example 1}

To demonstrate the advantage of the proposed prefix, first we use a simple wireless channel with only two paths $H(z)=\left(1+z^{-1}\right) / \sqrt{2}$. Then $H[k]=\left(1+e^{-j 2 \pi k / N}\right) / \sqrt{2}$. Considering an OFDM system with $N=64$, we notice that the 32 th subcarrier has a spectral null, since $H[32]=0$. This leads to an error floor of $1 /(2 \times 64)=0.0078125$. 


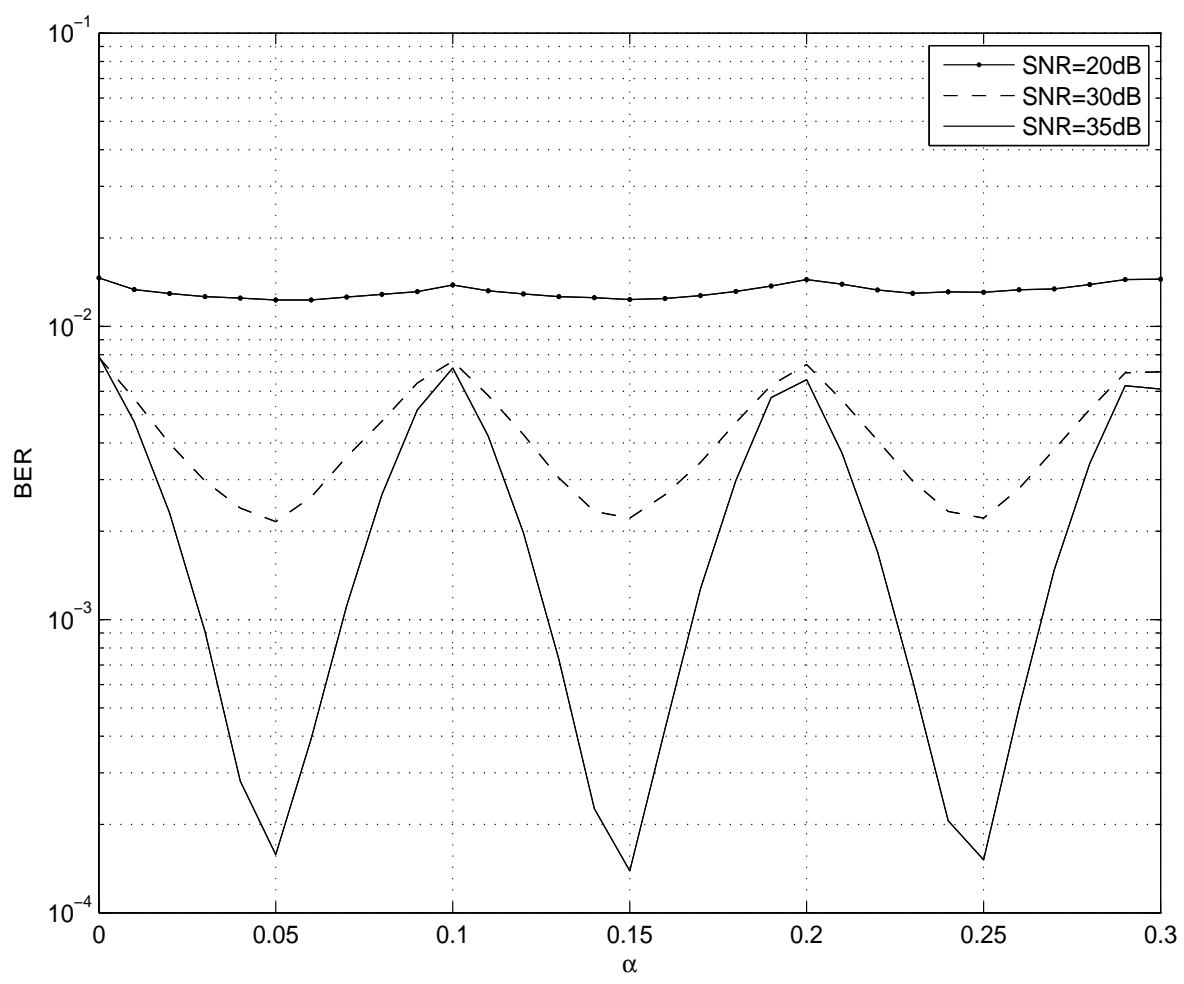

Figure 3: BER performance for different $\alpha$ values.

The use of a cyclic prefix scheme means that $\alpha$ is $2 \pi / N \approx 0.09817477$. But, this frequency domain shift only moves the existing spectral null from one subcarrier to another and the system error floor remains unchanged. This simple yet useful example illustrates some difficulties of the cyclic prefix.

To develop some intuition about the significance of the optimum value of $\psi=e^{j \alpha}$, the bit error rate (BER) performance of the proposed system is shown in Fig. 3 for different values of $\alpha$ at selected values of $E_{b} / N_{0} \in\{20,30,35\} \mathrm{dB}$. It is shown that the chosen $\psi$ plays an important role in the BER performance of the proposed system. Moreover, it is observed that the objective function is unimodal over the range $\alpha \in[0,2 \pi / N] \simeq[0,0.1]$, for $N=64$. Also, its minimum values are numerically found at points of the form $\psi=e^{j(2 m+1) \pi / N}$, where $m$ is an integer. This latter fact is analytically discussed below. The optimum value numerically obtained is $\alpha^{*} \approx 0.05$. This value is in agreement with the overall behavior of the channel frequency response. In fact, given that there is a single spectral null, which is located at subcarrier $k=N / 2$, it is expected that the best way to move the null away from the affected subcarrier is to place it between two adjacent subcarriers. This can be accomplished by a minimum frequency shift of $\mid \omega_{k}-$ $\omega_{k \pm 1} \mid / 2=\pi / N \approx 0.049087385$. In this case, the proposed prefix transforms the linear convolution into a skew-circular convolution. Considering the discussed class of equivalence, any frequency shift of the form $\alpha^{*}=\pi / N+2 \pi m / N=(2 m+1) \pi / N$, for any integer $m$, is adequate for our purposes.

Finally, the proposed system is compared with CP-OFDM and ZP-OFDM in Fig. 4 for the following parameters: 4-QAM modulation, $N=64$, and $K=16$. It is seen that CP-OFDM has an error floor at about $10^{-2}$. It is also seen that using the proposed prefix leads to a substantial performance advantage. In 


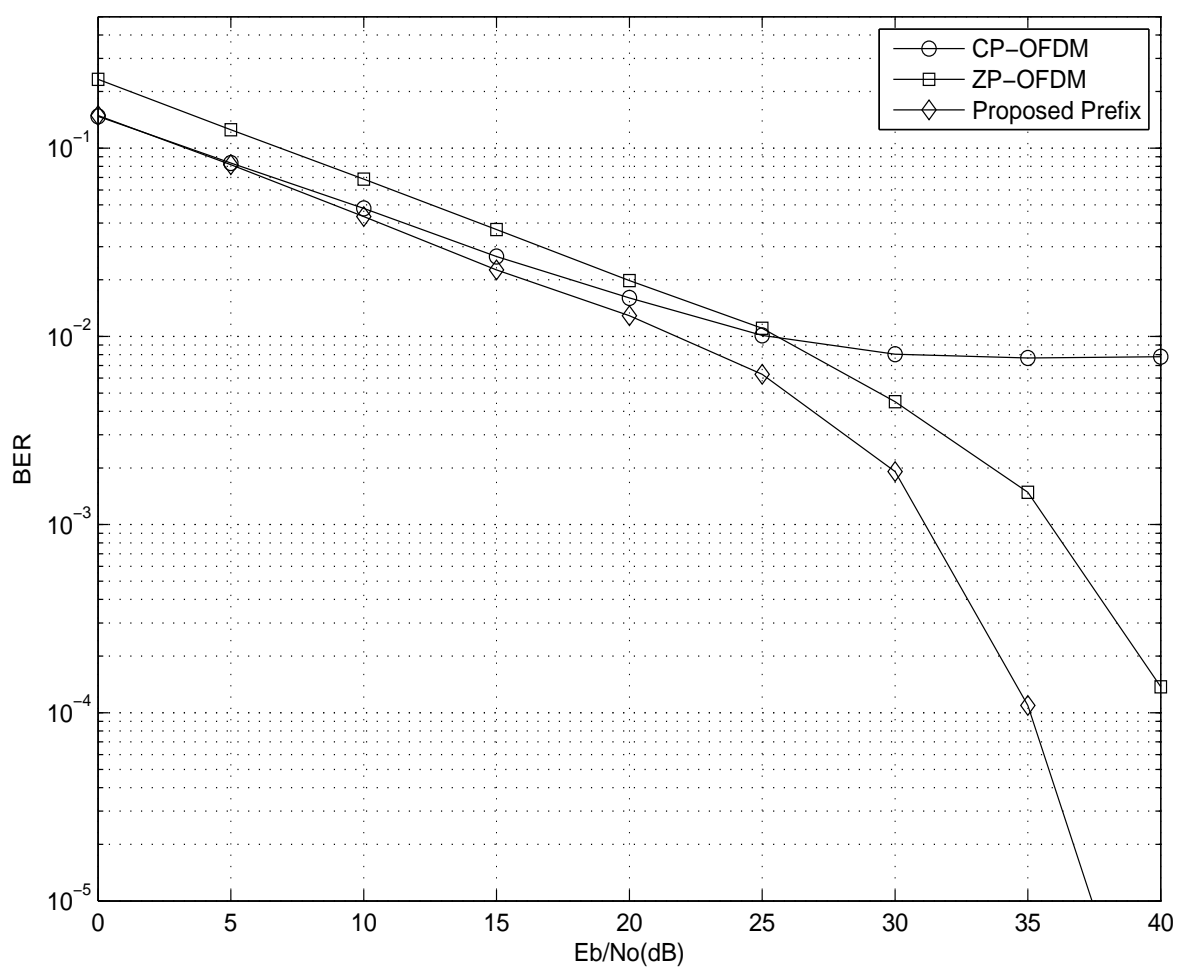

Figure 4: Comparison of bit error rates for OFDM with cyclic prefix, OFDM with zero-padding, and the proposed prefix.

particular, it outperforms ZP-OFDM by about $5 \mathrm{~dB}$ at BER $=10^{-4}$ (in addition to having a much lower computational complexity).

\subsection{EXAmPLE 2}

Now we consider a system appropriate for outdoor wireless communications with a $5 \mathrm{MHz}$ bandwidth, divided into $N=512$ tones. If we let the prefix length to be $K=64$, the total OFDM symbol would consist of $N+K=576$ samples. This implies a total symbol period of $115.2 \mu \mathrm{s}$, of which $12.8 \mu \mathrm{s}$ is allocated to the prefix. The OFDM frame has a time duration $16.12 \mathrm{~ms}$, consisting of 20 slots, each $0.806 \mathrm{~ms}$. Each slot consists of seven OFDM symbols. In the case of block-type channel estimation, the first OFDM symbol of each slot is allocated as pilot tones. The QPSK modulation format is employed. Additionally, the wireless channel between the transmitting antenna and the receiver antenna is modeled according to realistic channel specifications determined by the COST-207 project [22, p. 82]. In the following, quasi-static channel is assumed unless otherwise specified. We considered the typical urban (TU) and the bad urban (BU) channel models with a 12-tap channel model. For the TU channel model the delay spread is approximately $1 \mu \mathrm{s}$ $(-10 \mathrm{~dB}$ on $5 \mu \mathrm{s})$. For the BU channel model the delay spread is approximately $2.5 \mu \mathrm{s}(-15 \mathrm{~dB}$ on $10 \mu)$. The power delay profile (PDP) of the channels that have been used is given in Fig 5 . 

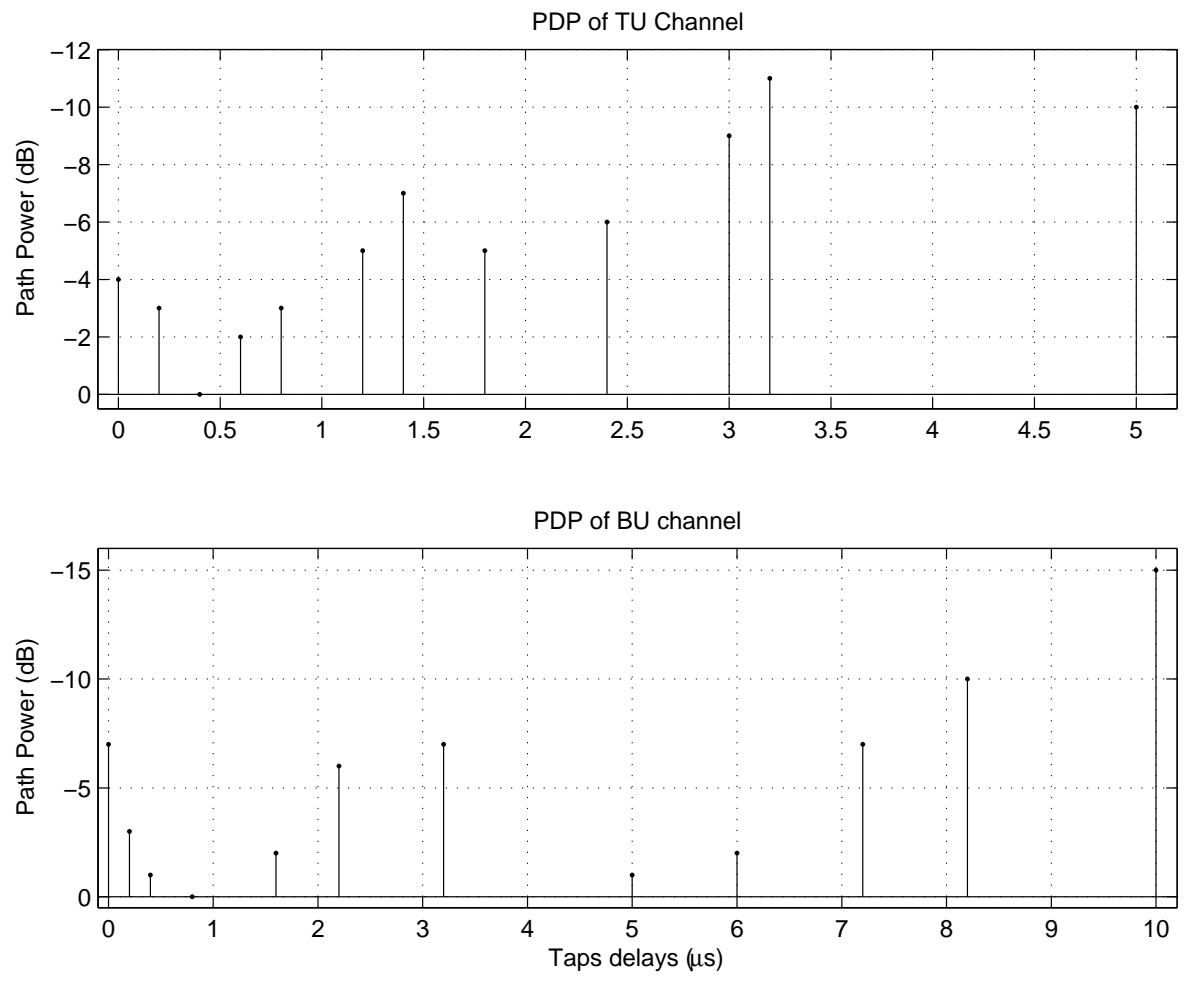

Figure 5: Power delay profiles of the COST 207 channels.

\subsubsection{PERFECT CSI}

Assuming that perfect CSI is available at the receiver, the BER of CP-OFDM and the proposed method is shown in Fig. 6

Considering TU channels, the proposed prefix gains about $7 \mathrm{~dB}$ over CP-OFDM at BER $=10^{-5}$. For $\mathrm{BU}$ channels, the gain at BER $=10^{-5}$ is about $9 \mathrm{~dB}$. These significant performance advantages intuitively can be explained as follows. Notice that longer delay spreads generally lead to more deep fades and spectral nulls. Therefore, BU channels have more deep fades than TU channels. For this reason, the the performance advantage of the proposed system is grater over BU channels. Therefore, the proposed prefix construction effectively mitigates the effects of multipath propagation.

Additionally, the performance difference between the proposed prefix and CP-OFDM increases as the SNR increases. This is especially noticeable for TU channels.

\subsubsection{Impact of Channel Estimation}

In the previous subsection perfect CSI was assumed.

In this subsection, we assume that the channel is not known exactly and channel estimation is necessary. Here, channel estimation is performed according to one of two approaches: (i) block-type estimation, which inserts pilot tones into all of subcarriers; or (ii) comb-type estimation, which uniformly inserts pilot tones into a OFDM symbol [23].

In our simulations we consider a quasi-static fading model, which allows the channel state to be constant 


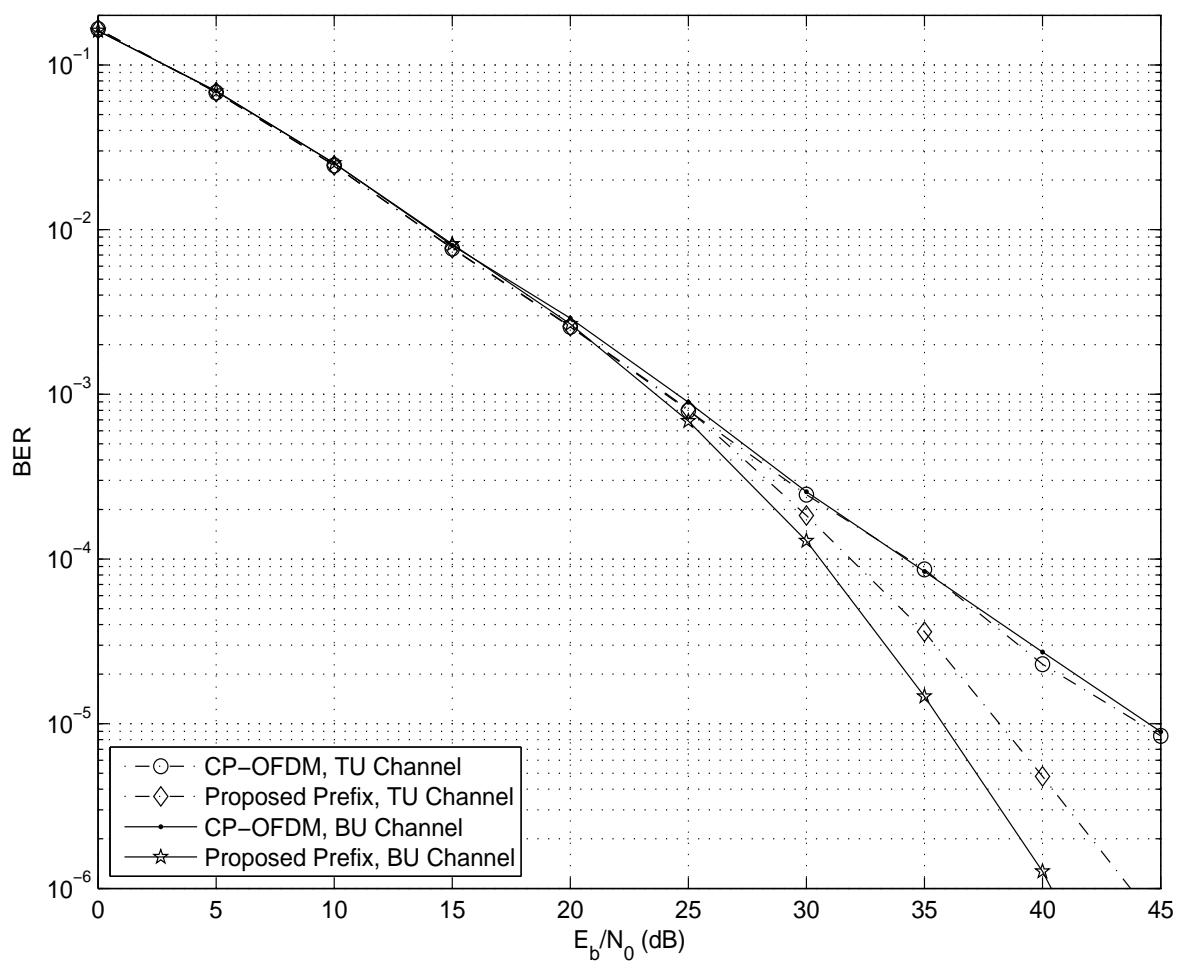

Figure 6: BER comparison of CP-OFDM and OP-OFDM over COST 207 channels (12 taps).

during the transmission of a block consisting of several OFDM symbols [24. In the case of block-type estimation, only the first received OFDM symbol was employed for the channel estimation and optimization procedures. Then the optimal $\psi$ was returned to the transmitter. Such optimized $\psi$ and the estimated channel response were reused at the receiver for the remaining OFDM symbols in the slot. The pilot-assisted channel estimation scheme that we use is based on least square (LS) techniques [25]. In the case of combtype estimation, the pilot insertion rate (PIR) was chosen to be 1:4. Channel estimation and optimization were performed for each OFDM symbol. The optimized values of $\psi$ were returned to the transmitter continuously. Equalization was done at the receiver by using the current $\psi$ values and estimated channel response. Additionally, a low-pass interpolation was also employed after LS estimation.

Imperfect CSI leads to some performance degradation for all systems compared to perfect CSI. Fig. 7 shows that for CP-OFDM there is a $3 \mathrm{~dB}$ loss at $\mathrm{BER}=10^{-4}$ in the case of block-type channel estimation. In contrast, the loss for the proposed system is only $0.8 \mathrm{~dB}$.

Therefore, the proposed prefix is more robust to channel estimation errors than the CP-OFDM. Similarly to the results in the previous subsection, the performance difference between the two considered systems increases as the SNR is increased, regardless of the type of channel estimation.

It is also of interest to investigate the performance of the proposed system in the presence of mobility. In Fig. 8, the BER of the CP-OFDM and the proposed system are shown for mobility of $20 \mathrm{~km} / \mathrm{h}$ where the Doppler frequency is equal to $44.44 \mathrm{~Hz}$. As expected, both systems have a significant performance loss for the block-type channel estimation. However, as expected, for both systems, comb-type estimation schemes 


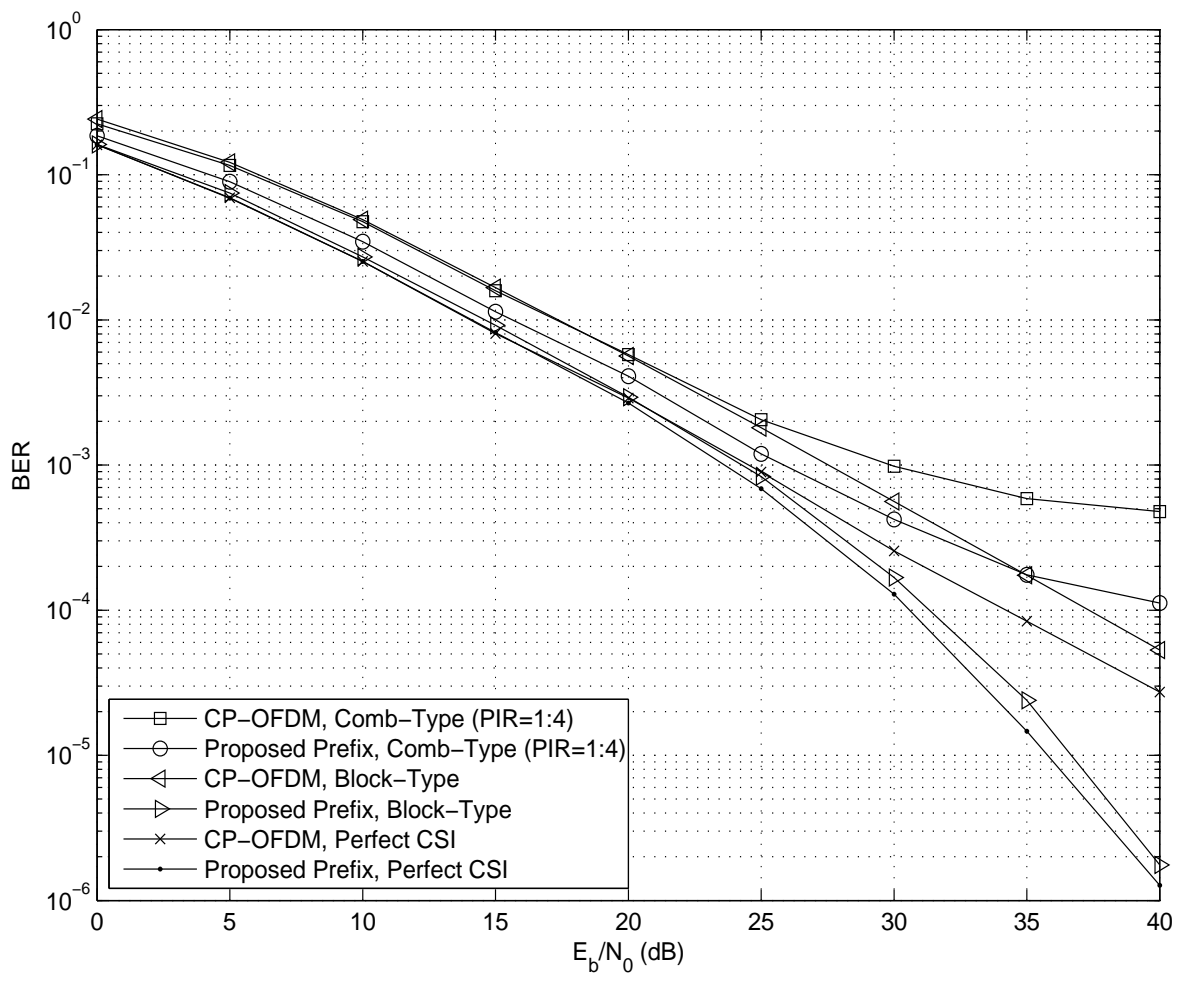

Figure 7: Impact of estimated channel impulse response on BER performance of the compared systems for BU channel (12 taps). 


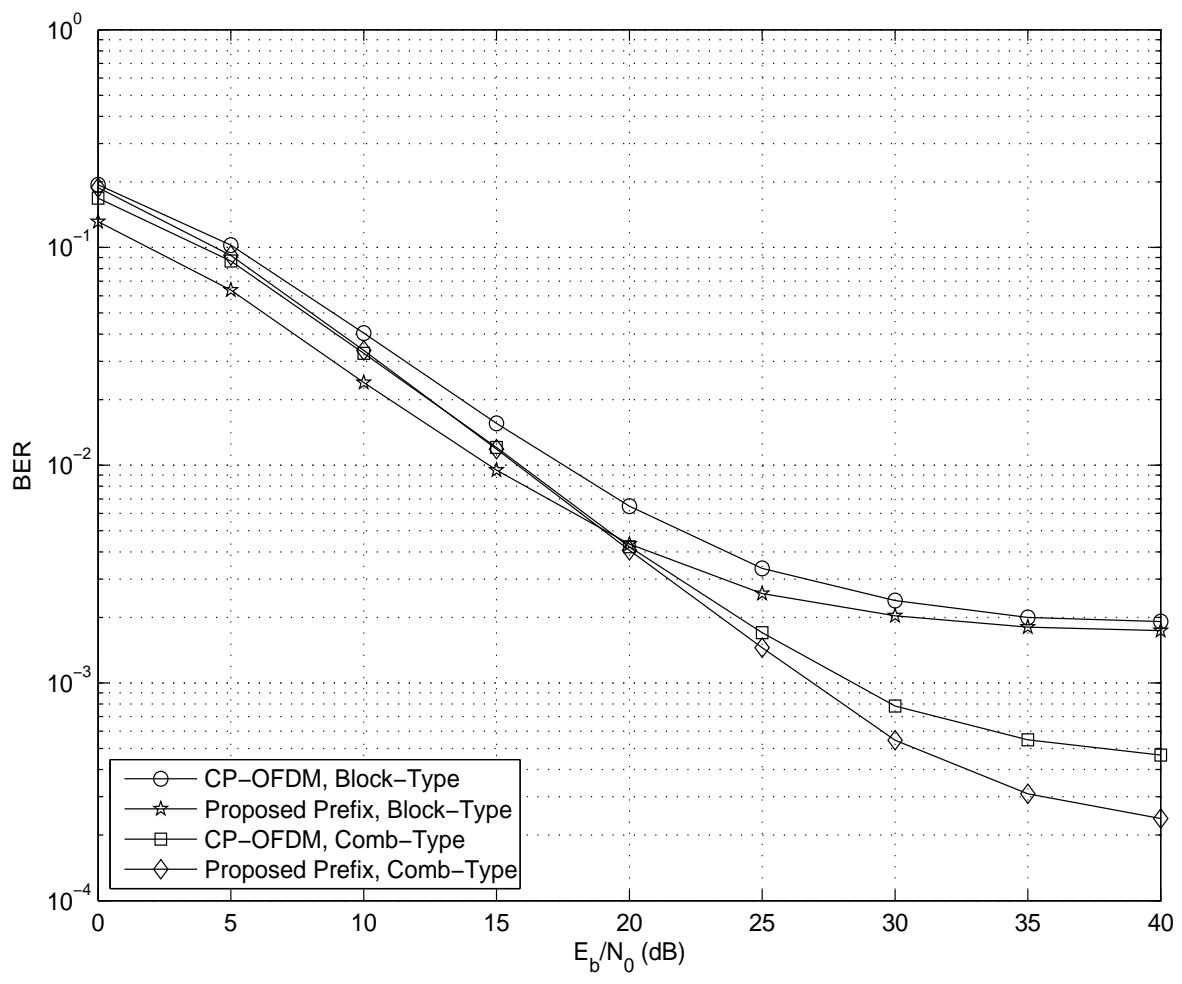

Figure 8: Impact of mobility on BER performance of the compared systems for BU channel (12 taps) at $20 \mathrm{~km} / \mathrm{h}$.

are more appropriate when there is mobility.

\section{Conclusions}

In this paper a new prefix for OFDM systems is proposed. The proposed system has the lowest bit error rate among the other prefix construction techniques, considering all other parameters identical. The prefix that is used is not random, it is determined using a computationally simple optimization routines. This prefix shifts the phases of the multipath components and effectively changes the wireless channel experienced by the OFDM system into a different channel. In particular, a wireless channel that has deep fades or spectral nulls is transformed into a channel with fades that are less deep or no spectral nulls. This property of the proposed system is equivalent to claiming that it ensures maximum diversity gain [2].

The reason for the performance advantages of the proposed technique can explained intuitively. For OFDM the probability of error is the average probability of error on all subcarriers, as in (11). In the presence of deep fades at some subcarriers, the probability of error for these subcarriers is large and will dominate. However, it is best if the probabilities of error for each subcarrier are identical or as much as possible similar. Then the probability of error of the system is minimized. This is what the new technique achieves. The simulation results indicate that the performance advantages are significant in all cases of interest. The performance improvement approaches $10 \mathrm{~dB}$ in the vicinity of $\mathrm{BER}=10^{-5}$ for $\mathrm{BU}$ channels. The technique is also superior in the presence of channel estimation errors and mobility. Also it is overall 
computationally simple and allows the use of cross-correlation or autocorrelation-based synchronization methods. The new method transforms the linear convolution into a convolution operation called generalized skew-circular convolution.

This research has further significance for orthogonal frequency-division multiple access (OFDMA) systems, in which each user experiences a different channel. In OFDMA systems with dynamic allocation, the base station informs the users about the assigned subcarriers [26]. In this case, due the dynamic variation of the number of active users, the optimization is computationally complex and the bandwidth consumed by the feedback channel is significant [27. Static allocation is simpler, but some of the assigned subcarriers may be experiencing deep fades. According to the proposed technique each user can apply an individual generalized prefix [28. Therefore, the system performance can be increased for both static and dynamic allocation systems. This is left as a topic for future research.

\section{ACKNOWLEDGMents}

This work was supported in part by Raytheon Corp., Fort Wayne, IN, through SERC grant 204135, by the Research Fund of Istanbul University under project YADOP-6265, by Conselho Nacional de Desenvolvimento Científico e Tecnológico (CNPq) and by FACEPE, Brazil.

\section{REFERENCES}

[1] I. P802.11n, Part 11: Wireless LAN Medium Access Control (MAC) and Physical Layer (PHY) Specifications: Amendment 5: Enhancements for Higher Throughput. IEEE-802.11 WG, Oct. 2009.

[2] Z. Wang and G. B. Giannakis, "Linearly precoded or coded OFDM against wireless channel fades?" in Proceedings of the Third IEEE Workshop Signal Processing Advances for Wireless Communications, Mar. 2001.

[3] X. G. Xia, "Precoded and vector OFDM robust to channel spectral nulls and with reduced cyclic prefix length in single transmit antenna systems," IEEE Transactions on Communications, vol. 49, no. 8, pp. 1363-1374, 2001.

[4] A. Scaglione, G. B. Giannakis, and S. Barbarossa, "Redundant filterbank precoders and equalizers Part I: Unification and optimal designs," IEEE Transactions on Signal Processing, vol. 47, no. 7, pp. 1988-2006, 1999.

[5] B. Muquet, Z. Wang, G. B. Giannakis, M. de Courville, and P. Duhamel, "Cyclic prefixing or zero padding for wireless multicarrier transmissions?" IEEE Transactions on Communications, vol. 50, no. 12, pp. 2136-2148, 2002.

[6] R. E. Blahut, Fast Algorithms for Signal Processing. Cambridge University Press, Aug. 2010.

[7] J. G. Andrews, A. Ghosh, and R. Muhamed, Fundamentals of WiMAX. Prentice Hall USA, 2007.

[8] M. Muck, M. de Courville, and P. Duhamel, "A pseudorandom postfix OFDM modulator-Semi-blind channel estimation and equalization," IEEE Transactions on Signal Processing, vol. 54, no. 3, pp. 1005-1017, 2006.

[9] L. Deneire, B. Gyselinckx, and M. Engels, "Training sequence versus cyclic prefix-a new look on single carrier communication," Communications Letters, IEEE, vol. 5, no. 7, pp. 292-294, 2001.

[10] M. Huemer, C. Hofbauer, and J. B. Huber, "Unique word prefix in SC/FDE and OFDM: A comparison," in IEEE GLOBECOM Workshops, Miami, USA, Dec. 2010, pp. 1296-1301.

[11] — - "Complex number RS coded OFDM with systematic noise in the guard interval,," in Signals, Systems and Computers (ASILOMAR), California, USA, Nov. 2010, pp. 1023-1028. 
[12] J. P. Coon and M. Sandell, "Designing unique words for near-optimal training in block transmission systems," European Transactions on Telecommunications, vol. 21, no. 1, pp. 13-22, 2010.

[13] H. Steendam and M. Moeneclaey, "Different guard interval techniques for OFDM: performance comparison," Multi-Carrier Spread Spectrum 200\%, pp. 11-24, 2007.

[14] A. V. Oppenheim and R. W. Schafer, Discrete-Time Signal Processing. Englewood Cliffs, NJ: Prentice Hall, 2009 .

[15] S. A. Martucci, "Symmetric convolution and the discrete sine and cosine transforms," IEEE Transactions on Signal Processing, vol. 42, no. 5, pp. 1038-1051, 1994.

[16] O. Ersoy, "Semisystolic array implementation of circular, skew circular, and linear convolutions," IEEE Transactions on Computers, vol. 100, no. 34, pp. 190-196, 1985.

[17] C. F. Gerald and P. O. Wheatley, Applied numerical analysis. Addison-Wesley Publishing Co, 2004.

[18] Z. Drezner, "On minimax optimization problems," Mathematical Programming, vol. 22, no. 1, pp. 227-230, 1982.

[19] Y. Rong, S. A. Vorobyov, and A. B. Gershman, "Adaptive OFDM techniques with one-bit-per-subcarrier channelstate feedback," IEEE Transactions on Communications, vol. 54, no. 11, pp. 1993-2003, 2006.

[20] L. Szczecinski, C. Gonzalez, and S. Aissa, "Exact expression for the BER of rectangular QAM with arbitrary constellation mapping," IEEE Transactions on Communications, vol. 54, no. 3, pp. 389-392, Mar. 2006.

[21] T. H. Cormen, C. E. Leiserson, R. L. Rivest, and C. Stein, Introduction to Algorithms. Prentice-Hall, 2001.

[22] K. L. Du and M. N. S. Swamy, Wireless Communication Systems: From RF Subsystems to 4G Enabling Technologies. Cambridge, U.K.: Cambridge University Press, 2010.

[23] S. Coleri, M. Ergen, A. Puri, and A. Bahai, "Channel estimation techniques based on pilot arrangement in OFDM systems," IEEE Transactions on Broadcasting, vol. 48, no. 3, pp. 223-229, Sep. 2002.

[24] A. Stamoulis, S. Diggavi, and N. Al-Dhahir, "Estimation of fast fading channels in OFDM," in Proceedings of the Wireless Communications and Networking Conference, WCNC2002, vol. 1, Mar. 2002, pp. 465-470.

[25] L. Tong, B. M. Sadler, and M. Dong, "Pilot-assisted wireless transmissions: general model, design criteria, and signal processing," IEEE Signal Processing Magazine, vol. 21, no. 6, pp. 12-25, Nov. 2004.

[26] F. Sun, M. You, and V. O. K. Li, "Dynamic subcarrier allocation for real-time traffic over multiuser OFDM systems," EURASIP Journal on Wireless Communicatons and Networking, vol. 2009, pp. 1-9, 2009.

[27] G. Song, Y. Li, and M. Inc, "Utility-based resource allocation and scheduling in OFDM-based wireless broadband networks," IEEE Communications Magazine, vol. 43, no. 12, pp. 127-134, 2005.

[28] A. K. F. Khattab and K. M. F. Elsayed, "Opportunistic scheduling of delay sensitive traffic in OFDMA-based wireless networks," in International Symposium on a World of Wireless, Mobile and Multimedia Networks, WoWMoM, 2006, p. 10. 